\title{
Predicting Asset Value Through Twitter Buzz
}

\author{
Xue Zhang ${ }^{\mathrm{a}, \mathrm{b}}$, Hauke Fuehres ${ }^{\mathrm{b}}$, Peter A. Gloor \\ ${ }^{a}$ Department of Mathematic and Systems Science, National University of Defense \\ Technology, Changsha, Hunan, P.R.China \\ ${ }^{\mathrm{b}}$ MIT Center for Collective Intelligence, Cambridge MA, USA
}

\begin{abstract}
This paper describes early work trying to predict financial market movement such as gold price, crude oil price, currency exchange rates and stock market indicators by analyzing Twitter posts. We collected Twitter feeds for 5 months obtaining a large set of emotional retweets originating from within the US, from which six public opinion time series containing the keywords "dollar\% ${ }_{t}$ ", " $\$ \%_{t}$ ", " gold $\%_{t}$ ", “oil\% $\%_{t}$ ", “ job\% $\%_{t}$ " and "economy $\%_{t}$ " were extracted. Our results show that these variables are correlated to and even predictive of the financial market movement. Except " $\$ \%_{t}$ ", all other five public opinion time series are identified by a Granger-causal relationship with certain market movements. It is demonstrated that daily changes in the volume of economic topic retweeting seem to match the value shift occurring in the corresponding market next day.
\end{abstract}

\section{Introduction}

"Prediction is difficult, especially when its about the future" - Niels Bohr

We human beings are always curious about the future. For thousands of years, people have been trying their best to predict what would happen next. Although most of these predictions turn out to be wrong, people never give up predicting, and are continuously trying to improve it. Weather forecasts, earthquake early warnings, stock market predictions etc. - predictions are an important part in everyday life. With the popularization of Internet and online social networking, this time-honored activity enters a new era.

Recently, a lot of research has been done on prediction with data from social networks and web searches. Gayo-Avello et al. [1] clearly pointed out that following what people are blogging about or what they are searching about can give us some intuition on the collective psyche and lead us to understand what is currently happening in society before it is actually happening. Sometimes people refer to this phenomenon as the "wisdom of the crowd", that is, taking into account the opinion of the society as a whole, instead of the opinion of the expert.

A group of researchers is applying this novel methodology to stock market prediction. Antweiler and Frank [2] determined correlation between activity in Inter- 
net message boards and stock volatility. Gilbert and Karahalios [3] used over 20 million posts from the LiveJournal website to create an index of the US national mood, which they call the Anxiety Index. They found that when this index rose sharply, the S\&P 500 ended the day marginally lower than is expected. Choudhury et al. [4] modeled contextual properties of posts in SVMs (support vector machines) and trained it with stock movement. The result shows about $87 \%$ accuracy in predicting the direction of the movement.

As one of the most popular social networking websites, Twitter is drawing more and more attention from researchers from different disciplines. There are several streams of research investigating the role of Twitter. One stream of research focuses on understanding its usage and community structure $[5,6,7,8]$. Other researchers are more interested in its prediction power and potential application in other areas. It has been demonstrated that by tracking tweet numbers related to certain topics, both box-office revenues of movies and political elections could be successfully forecasted $[9,10]$. Also, Twitter has been used in tracking the spread of epidemic disease [11].

Twitter buzz was also employed in predicting the stock market movement. By analyzing the sentiment of a random sample of tweets, Bollen et al. [12] found that public mood can be used to predict the stock market. Furthermore, stock-related tweets with a specific hashtag "\$" were collected and studied in detail in [13], where it was found that these tweets contain valuable information that is not fully incorporated in current market indicators. In previous work [14], we also presented very preliminary results that the number of emotional tweets, which contain words such as "hope", "fear" or "worry" correlated with stock market indicators. In this paper, further tests and analysis to predict valuation of tradable assets will be described.

The rest of the paper is organized as follows. In section 2, we present our Twitter dataset and financial market dataset, laying out how we constructed the public opinion time series. Section 3 discusses the method of determining correlation between Twitter buzz and market movement and presents the results, which are followed by discussion and future work in section 4 .

\section{Data}

Twitter is a worldwide popular website, which offers a social networking and microblogging service, enabling its users to update their status in tweets, follow the people they are interested in, retweet others' posts and even communicate with them directly. Since it launched in 2006, its user base has been growing exponentially. As of June 2011, it is estimated to have 200 million users, generating 190 million tweets a day and handling over 1.6 billion search queries per day. The rising popularity of twitter gives us a novel way of capturing the collective mind up to the last minute. In this section, we introduce the datasets that form the basis of the work described in this paper. 


\subsection{Twitter Data Collection}

We collected a large set of tweets submitted to Twitter in the period from November 15, 2010 to April 20, 2011. In order to get a better picture of the opinion and emotional state of the US investors, we only filter for emotional retweets that come from the United States. In other words, all the data we collected meets the following conditions:

Retweets only. Structurally, retweeting is the Twitter-equivalent of email forwarding where users post messages originally posted by others. As an integral part of the Twitter experience, the retweeting phenomenon has been explicitly studied in prior research [7]. It is generally believed that the more a topic is being picked up and retweeted by others, the more it is relevant and widely recognized. Although there is no universally agreed-upon syntax for retweeting, "RT @user message" is the prototypical formulation where the referenced user is the original author and message is the original tweet's content, therefore we choose "RT @" as our indicator of retweets.

Containing the emotion words "hope", "fear" or "worry". Emotional state greatly influences human decisions, which obviously include the appropriate choice of an investment strategy $[15,16,17,18,19]$. When people are pessimistic or uncertain about their future, they will be more cautious to invest and trade. Therefore capturing the collective mind - especially people's mood - becomes one possible way to predict the future. To be consistent with and further test previous work, we only take into account the retweets that contain the words "hope", "fear" or "worry", because we had found in earlier work [14] that these words are excellent indicators of emotion-laden tweets.

Originating from the US. The goal of this paper is to analyze whether Twitter buzz can be helpful in forecasting selected economic indicators of the US economy. For the purpose of better capturing the opinion and emotional state of the US population, we intentionally limit the targeted tweets to the ones originating from within the continental United States without Alaska. Tweets were collected within four 2000-kilometers circles with centers in Pittsburg, Atlanta, Las Vegas and Boise respectively. As Figure 1 shows, these circles cover the contiguous United States and parts of Canada and Mexico.

Over the duration of five months, 3,809,437 retweets posted by approximately 961,000 users were collected and each tweet has a unique identifier, time of submission and the textual content. Table 1 summarizes the daily number of retweets related to each emotional word. As we can see, the daily retweet rate of each emotion word is highly variable, for example, the hope-retweets range from 6453 to 34805 per day. Even more interestingly, the number of hope-retweets is much higher than the fear or worry ones, almost six times on average, which might suggest that people prefer using optimistic words when they express their feelings, even when they are worrying or in fear. 


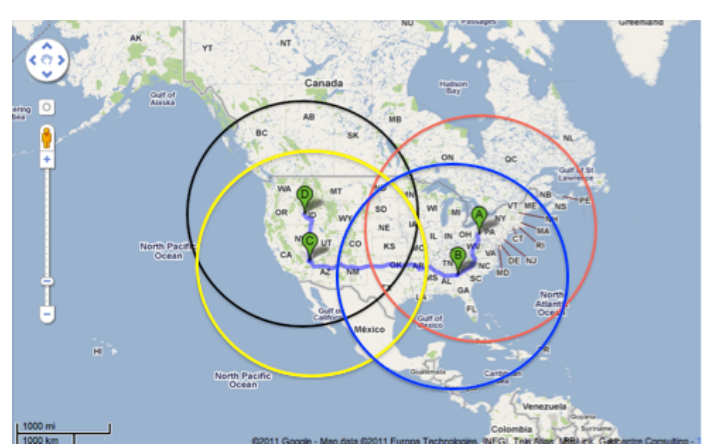

Fig. 1 Geographical origin of Twitter data: A is Pittsburg, B is Atlanta, C is Las Vegas and D is Boise

Table 1 Daily number of emotional retweets

\begin{tabular}{llll}
\hline & Average per day & Min per day & Max per day \\
\hline Hope-retweet\# & 20613 & 6453 & 34805 \\
Fear-retweet\# & 3710 & 853 & 7555 \\
Worry-retweet\# & 3653 & 1071 & 7397 \\
Total\# & 27977 & 11395 & 46209 \\
\hline
\end{tabular}

\subsection{Generating public opinion time series}

In this section, we further discuss how to extract posts in regard to economic topics from our emotional retweets dataset. As twitter users can share only short textual messages with no more than 140 characters per post, there is always only one topic in one tweet. No matter if it is a piece of news announcing the death of Osama bin Laden or a conversation between two friends talking about the wonderful birthday party, owing to the length limitation, the tweet stays on the same topic. Thus the main theme of the whole tweet can usually be subsumed by one or two keywords.

Inspired by this property, a list of words related to economy was selected as a clue for economic tweets. This list of keywords includes "dollar", “\$”, "gold" "oil", "job" and "economy". Then we measured collective opinion on each day by simply counting how many retweets contain these words. As the total number of retweets varies highly from day to day, a normalized number was chosen as a measurement of public opinion on day $t$. For example, we counted the number of retweets containing the word "dollar" and normalized it by the total retweet number on the same day $t$, this normalized retweet number is listed as "dollar $\%_{t}$ ". Figure 2 below illustrates all 6 public opinion time series. 


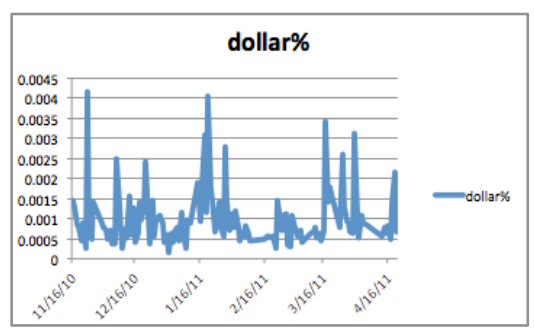

(a) dollar $\%$

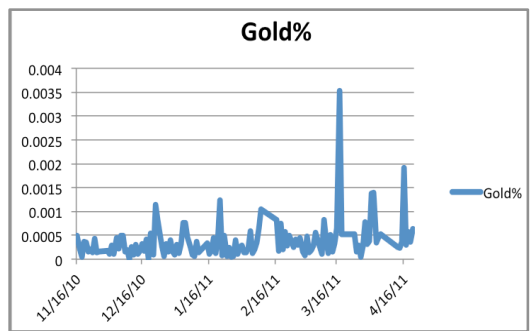

(c) gold $_{t}$

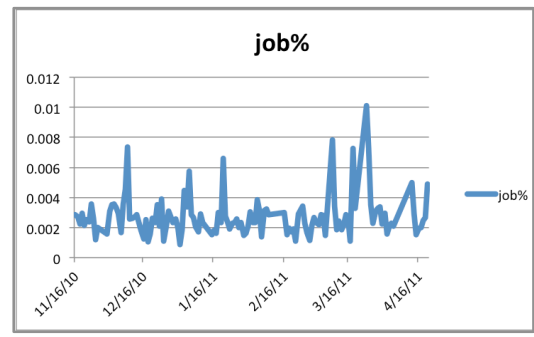

(e) $j o b \%$

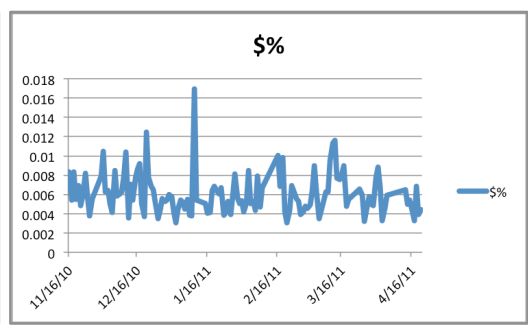

(b) $\$ \%$,

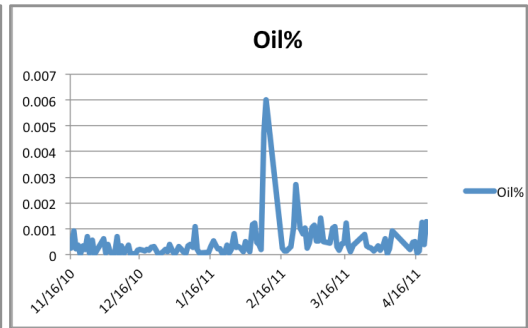

(d) oil $\%_{t}$

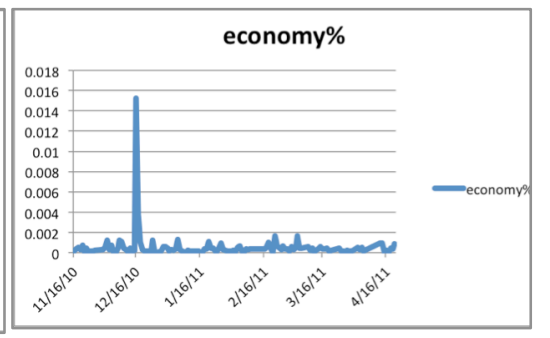

(f) economy $\%$

Fig. 2 Public opinion time series

\subsection{Market Data}

In this section, we look at different categories of assets including the gold price, crude oil price, currency exchange rates and stock market indicators. For our analysis we have taken the daily price of gold (dollars per ounce), WTI Cushing crude oil price (dollars per barrel), currency exchange rates (USD/CHF), Dow Jones Industrial Average (DJIA), NASDAQ and S\&P 500 all collected during the same period as the Twitter data. 


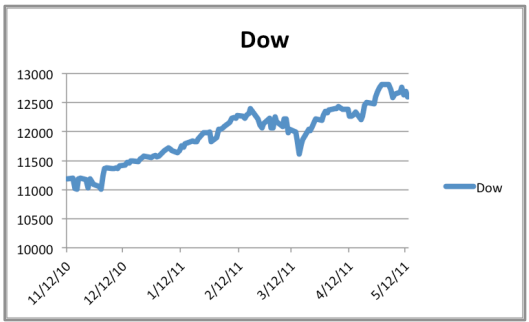

(a) DJIA

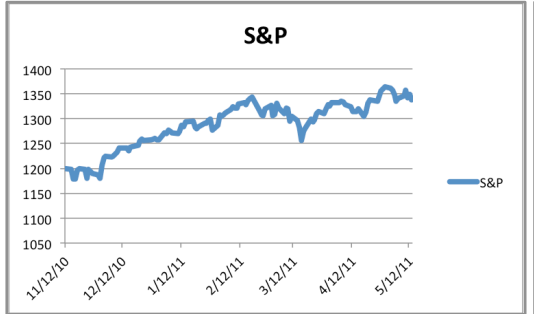

(c) $S \& P 500$

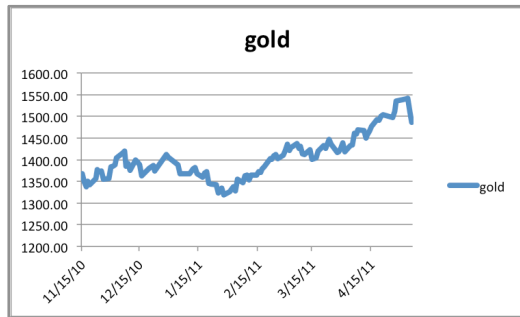

(e) gold price (dollars per ounce)

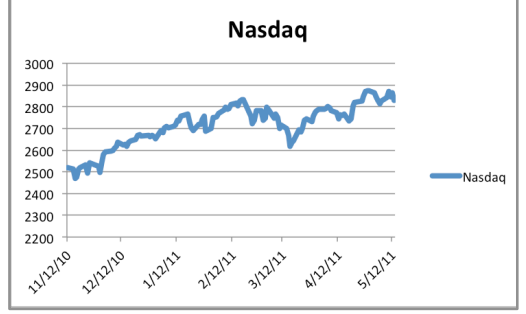

(b) NASDAQ

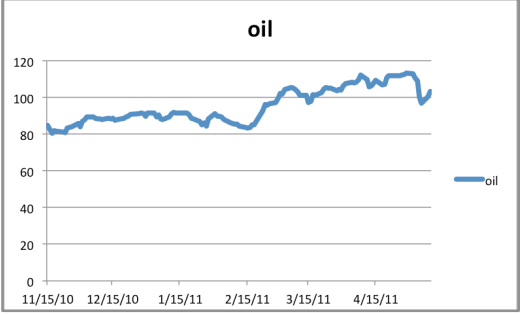

(d) oil price (dollars per barrel)

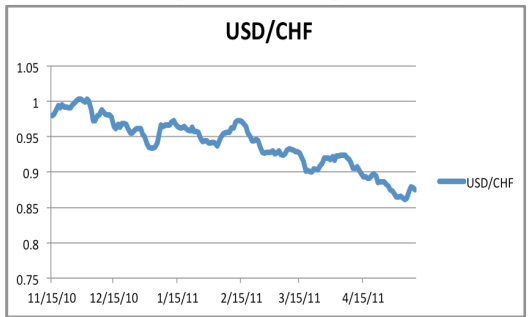

(f) currency exchange rates (USD/CHF)

Fig. 3 Market time series

From Figure 3, we can see that although there is much fluctuation, the overall trend in this period of stock market, crude oil and gold price is all up. However, in contrast, the exchange rate of USD to CHF declined almost $10 \%$ at the same time. Obviously, all these market time series are non-stationary. To meet the requirement of stationarity in time series analysis, data are processed in the following way. Taking DJIA as an example, the stock movement at a day $t$ is defined as the normalized change in stock close price from the past day, which can be expressed as

$$
D_{t}=\frac{D J I A_{t}-D J I A_{t-1}}{D J I A_{t-1}},
$$

where $D J I A_{t}$ is the close price of day $t$. Similarly, we determine the other independent variables. Using these new relative variables, we not only can tell the 
change direction of the market which is indicated by the sign of the number, but alsoalso measure how much it changed compared to the previous day.

$$
\begin{array}{ll}
N_{t}=\frac{N A S D A Q_{t}-N A S D A Q_{t-1}}{N A S D A Q_{t-1}} & \text { (2) } S_{t}=\frac{S \& P_{t}-S \& P_{t-1}}{S \& P_{t-1}} \\
O_{t}=\frac{\text { Oil }_{t}-\text { Oil }_{t-1}}{\text { Oil }_{t-1}} & \text { (4) } G_{t}=\frac{\text { Fold }_{t}-\text { Gold }_{t-1}}{\text { oold }_{t-1}} \\
U_{t}=\frac{U S D_{t}-U S D_{t-1}}{U S D_{t-1}} & \text { (6) }
\end{array}
$$

\section{Methods and Results}

\subsection{Correlation between public opinion and market time series}

To obtain a first indication whether the Twitter information might help forecast the asset value, we analyzed the correlation between the two time series. Tables 2 to 4 illustrate correlation coefficients between market movement on day $t$ and Twitter buzz of day $t-i(i=1,2,3)$ separately.

In Table 2, we observe a relatively strong correlation between stock market return and "dollar $\%_{t-1}$ " $\left(r=0.308^{* *}, 0.203\right.$ and $0.259^{*}, p$-value $=0.004,0.058$ and 0.015 ). In addition, not only " oil $\%_{t-1}$ " but also "economy $\%_{t-1}$ " is strongly correlated with oil price changes of day $t\left(r=0.295^{* * *}\right.$ and $0.214^{*}, p$-value $=0.006$ and 0.046). Even more interestingly, we found that the correlation between " gold $\%_{t-1}$ " and $G_{t}$ is weak, but " gold $\%_{t-1}$ " is significantly correlated with $U_{t}\left(r=0.213^{*}\right.$, $p$-value $=0.016)$, indicating a relationship between the gold price and the strength of the US dollar. Furthermore, it is worth noticing that all the correlation coefficients mentioned above are positive, which implies that an increase in economic topic retweeting seems to indicate an increase in the value of the corresponding asset on the next market day.

In contrast, the relationships between market movement and time series " $\$ \%$ " and " job\%" are not that significant in this period. Additionally, the Twitter buzz of two or three days before seems to have less influence on the market movement of day $t$ (see Tables 3 and 4). 
Table 2 Correlation coefficient between market movement and Twitter buzz 1 day before

\begin{tabular}{lllllll}
\hline & $D_{t}$ & $N_{t}$ & $S_{t}$ & $O_{t}$ & $G_{t}$ & $U_{t}$ \\
\hline${\text { dollar } \%_{t-1}}$ & $.308^{* *}$ & .203 & $.259^{*}$ & .012 & -.112 & -.055 \\
$\$ \%_{t-1}$ & .108 & .062 & .116 & .004 & -.080 & -.122 \\
${\text { gold }{ }_{t-1}}$ & .122 & .055 & .088 & -.034 & -.053 & $.213^{*}$ \\
oil\% $_{t-1}$ & .022 & .018 & .054 & $.295^{* *}$ & .108 & .072 \\
job\% $_{t-1}$ & -.035 & .000 & -.013 & -.165 & -.203 & .167 \\
economy $_{t-1}$ & -.142 & -.186 & -.147 & $.214^{*}$ & -.011 & -.021 \\
\hline
\end{tabular}

Table 3 Correlation coefficient between market movement and Twitter buzz 2 day before

\begin{tabular}{lcccccc}
\hline & $D_{t}$ & $N_{t}$ & $S_{t}$ & $O_{t}$ & $G_{t}$ & $U_{t}$ \\
\hline${\text { dollar }{ }_{t-2}}$ & .106 & .040 & .065 & -.148 & -.078 & .122 \\
$\$ \%_{t-2}$ & .040 & .025 & .033 & -.099 & .077 & -.131 \\
gold $_{t-2}$ & -.032 & -.013 & -.041 & .020 & .089 & .064 \\
oil\% $_{t-2}$ & .004 & -.039 & -.019 & .201 & -.004 & -.123 \\
job\% $_{t-2}$ & .094 & .101 & .108 & -.151 & -.116 & .081 \\
economy $_{t-2}$ & -.073 & -.068 & -.030 & .121 & .020 & -.039 \\
\hline
\end{tabular}

Table 4 Correlation coefficient between market movement and Twitter buzz 3 day before

\begin{tabular}{lcccccc}
\hline & $D_{t}$ & $N_{t}$ & $S_{t}$ & $O_{t}$ & $G_{t}$ & $U_{t}$ \\
\hline${\text { dollar } \%_{t-3}} .018$ & .010 & .026 & -.013 & $-.273^{*}$ & -.088 \\
$\$ \%_{t-3}$ & -.198 & -.179 & -.176 & -.109 & -.048 & -.142 \\
${\text { gold } \%_{t-3}}$ & .020 & .024 & .007 & -.033 & .030 & .133 \\
oil\% $_{t-3}$ & .033 & .077 & .069 & .039 & -.039 & -.017 \\
job\% $_{t-3}$ & .126 & .156 & .130 & -.132 & -.141 & .093 \\
economy\% $_{t-3}$ & -.086 & -.025 & -.029 & .031 & .152 & -.118 \\
\hline
\end{tabular}




\subsection{Granger-causality Analysis}

In this section we apply Granger causality analysis to the daily time series of public opinion vs. financial market movement. Granger causality is a statistical concept of causality that is based on prediction. According to Granger causality, if a signal $X$ "Granger-causes" (or "G-causes") a signal $Y$, then past values of $X$ should contain information that helps predict $Y$ above and beyond the information contained in past values of $Y$ alone. Its mathematical formulation is based on linear regression modeling of stochastic processes (Granger 1969). It is noteworthy that in spite of its name, Granger causality is not sufficient to imply true causality. If both $X$ and $Y$ are driven by a common third process with different lags, $X$ might erroneously be believed to "Granger-cause" $Y$. However, in our project, we are not testing the actual causation but simply whether one variable provides predictive information about the other one or not.

Granger causality requires that the time series have to be covariance stationary, so an Augmented Dickey-Fuller test has been done first, in which the null hypothesis $H_{0}$ of non-stationarity was rejected at the 0.05 confidence level. Again, all Twitter buzz and market movement time series were verified to be stationary.

To test whether public opinion time series "Granger-cause" the changes in financial market valuation, two linear regression models were applied as shown in equations 7 and 8 . The first model $\left(M_{1}\right)$ uses only $n$ lagged values of market data to predict $Y_{t}$, while the second model $\left(M_{2}\right)$ also includes the lagged value of public opinion time series, which are denoted by $X_{t-1}, \ldots, X_{t-n}$. In order to find an appropriate number of lags, we set the lag parameter $n$ equal to 1,2 and 3 separately.

$$
\begin{aligned}
& M_{1}: Y_{t}=\alpha+\sum_{i=1}^{n} \beta_{i} Y_{t-i}+\varepsilon_{t} \\
& M_{2}: Y_{t}=\alpha+\sum_{i=1}^{n} \beta_{i} Y_{t-i}+\sum_{j=1}^{n} \gamma_{j} X_{t-j}+\varepsilon_{t}
\end{aligned}
$$

After establishing the linear regression equations, a statistics $f$ is defined as

$$
f=\frac{\left(\frac{S S R_{1}-S S R_{2}}{n}\right)}{\left(\frac{S S R_{2}}{m-2 n-1}\right)}
$$

where $S S R_{1}$ and $S S R_{2}$ are the two sum of squares residuals of equations 7 and $8 ; m$ is the number of observations. Theoretically, $f \sim F(n, m-2 n-1)$. Thus, the ques- 
tion whether $X$ "Granger-causes" $Y$ could be solved by simply checking the $p$ values.

From Table 5 we can easily draw the conclusion that Twitter buzz indeed has some information that can be used in predicting financial market movement. We observe that "dollar\% $\%_{t}$ " has the highest Granger causality relation with stock market return, especially with the DJIA return ( $p$-value $<0.01$ when $n=1$ and remains significant when $n=2$ and 3 ). Also, the "oil\% $t$ " time series "Grangercauses" the changes in oil price ( $p$-value is always less than 0.05 when lag varies from 1 to 3 days). The other two predictive variables are " gold $\%_{t}$ " and " job $\%_{t}$ ", which have Granger causality relation with $U_{t}$ and $G_{t}$ separately. However, the most interesting aspect is that the "gold $\%_{t}$ " time series failed in explaining the price change in the gold market, but could help predict the currency exchange rates (USD/CHF). We speculate that currency fluctuation, and the underlying lack in confidence in the national economy influence the eagerness of buyers to invest into the "safe haven" gold.

Table 5 Statistical significance ( $p$-value) of bivariate Granger causality correlation between Twitter buzz and financial market movement ( $p$-value $<0.05$ : *, p-value $<0.01$ : **)

\begin{tabular}{|c|c|c|c|c|c|c|c|}
\hline & Lag & $D_{t}$ & $N_{t}$ & $S_{t}$ & $O_{t}$ & $G_{t}$ & $U_{t}$ \\
\hline \multirow{3}{*}{ dollar $\%_{t}$} & $n=1$ & $.0039 * *$ & $.0494 *$ & $.0165^{*}$ & .9588 & .2877 & .3131 \\
\hline & $n=2$ & $.0203 *$ & .2405 & .124 & .3174 & .3301 & .1014 \\
\hline & $n=3$ & $.0272 *$ & .3869 & .1803 & .4251 & .0527 & .0802 \\
\hline \multirow{3}{*}{$\$ \%_{t}$} & $n=1$ & .3309 & .5538 & .2966 & .9033 & .4887 & .2315 \\
\hline & $n=2$ & .9919 & .9864 & .9849 & .7672 & .6766 & .357 \\
\hline & $n=3$ & .2369 & .3818 & .3967 & .8694 & .7855 & .3395 \\
\hline \multirow{3}{*}{${\text { gold } \%_{t}}$} & $n=1$ & .286 & .5908 & .4459 & .7685 & .5943 & $.0053 *$ \\
\hline & $n=2$ & .3047 & .7991 & .572 & .8669 & .7787 & $.0306^{*}$ \\
\hline & $n=3$ & .3267 & .8883 & .7072 & .9317 & .9964 & .0518 \\
\hline \multirow{3}{*}{$\mathrm{oil}_{t}$} & $n=1$ & .9036 & .7989 & .6828 & $.0164 *$ & .2359 & .6347 \\
\hline & $n=2$ & .8657 & .4439 & .7809 & $.0096 * *$ & .3672 & .6156 \\
\hline & $n=3$ & .9102 & .5801 & .864 & $.0225^{*}$ & .3668 & .3782 \\
\hline \multirow{3}{*}{$j o b \%_{t}$} & $n=1$ & .7542 & .9812 & .9222 & .1668 & $.0413^{*}$ & .1167 \\
\hline & $n=2$ & .2322 & .3358 & .204 & .3986 & .194 & .3919 \\
\hline & $n=3$ & .4879 & .5408 & .4544 & .3697 & .0896 & .6271 \\
\hline
\end{tabular}




\begin{tabular}{llllllll}
\hline & $n=1$ & .1978 & .0819 & .183 & .0558 & .8752 & .591 \\
economy\% $_{t}$ & $n=2$ & .6916 & .297 & .5575 & .1293 & .9446 & .7235 \\
& $n=3$ & .6592 & .4267 & .6904 & .1896 & .4743 & .3417 \\
\hline
\end{tabular}

\section{Discussions}

In this paper, we investigated the relationship between Twitter buzz and financial market movement. Our results statistically show that public opinion measured from large-scale collection of emotional retweets is correlated to and even predictive of the financial market movement. Except " $\$ \%_{t}$ ", all other five public opinion time series are identified in a Granger-causal relationship with selected asset valuation movements. The changes in the volume of economic topic retweeting seems to match the value shift occurring in corresponding next market day.

However, there are still a number of important factors not acknowledged in our analysis to be studied in future work. First, unlike the prior work of [12] and [13], when we extracted the public opinion from Twitter, we neither constrained our data to those stock-related tweets which have a specific hashtag nor use sentiment analysis tools to measure the public mood from a random sample of tweets. We chose a few keywords to identify the emotional retweets talking about economic activity, then use volume change to track the public opinion. This method is simple and useful. It however does not linguistically analyze the content of tweets, which might offer additional valuable information. Advanced sentiment analysis could be employed in future work to improve our results. Second, the analyzing methods we used in this paper, both the correlation and Granger causality analysis, are based on the assumption that the relation between variables is linear, which is hardly satisfied for financial market movement. More advanced tools that can better characterize the non-linear relationship between variables, such as Neural Networks and Support Vector Machines, should also be explored in future work.

\section{Reference}

1. Gayo-Avello, D., Metaxas, P.T. \& Mustafaraj. E. (2011). On the Unpredictability of Elections using Social Media Data. Interdisciplinary Workshop on Information and Decision in Social Networks, MIT.

2. Antweiler, W., \& Frank, M.Z. (2004). Is All That Talk Just Noise? The Information Content of Internet Stock Message Boards. Journal of Finance, 59(3), 1259-1294.

3. Gilbert, E., \& Karahalios, K. (2010). Widespread Worry and the Stock Market. In Proceedings of the $4^{\text {th }}$ International AAAI Conference on Weblogs and Social Media (ICWSM). 
4. Choudhury, M.D., Sundaram, H., John, A., \& Seligmann, D.D. (2010). Can Blog Communication Dynamics be Correlated with Stock Market Activity? In Proceedings of the $9^{\text {th }}$ ACM Conference on Hypertext and Hypermedia. doi:10.1145/1379092.1379106.

5. Java, A., Song, X., Finin, T., \& Tseng, B. (2007) Why We Twitter: Understanding Microblogging Usage and Communities. In Proceeding of $9^{\text {th }}$ WebKDD and $1^{\text {st }}$ SNA-KDD workshop on web mining and social network analysis. doi:10.1145/1348549.1348556.

6. Huberman, B.A., Romero, D.M., \& Wu F. (2009). Social networks that matter: Twitter under the microscope. First Monday, 14(1).

7. Boyd, D., Golder, S., \& Lotan, G. (2010). Tweet, Tweet, Retweet: Conversational Aspects of Retweeting on Twitter. In Proceeding of $43^{\text {rd }}$ Hawaii International Conference on System Sciences (HICSS). doi: 10.1109/HICSS.2010.412.

8. Wu, S., Hofman, J.M., Mason, W.A., \& Watts, D.J. (2011). Who says What to Whom on Twitter. In Proceedings of the $20^{\text {th }}$ International Conference on World Wide Web. doi:10.1145/1963405.1963504.

9. Asur, S., \& Huberman, B.A. Predicting the Future With Social Media. (2010). In Proceedings of the 2010 IEEE/WIC/ACM International Conference on Web Intelligence and Intelligent Agent Technology. doi: 10.1109/WI-IAT.2010.63.

10. Tumasjan, A., Sprenger, T.O., Sandner P.G., \& Welpe I.M. (2010). Predicting Elections with Twitter: What 140 Characters Reveal about Political Sentiment. In Proceedings of the $4^{\text {th }}$ International AAAI Conference on Weblogs and Social Media (ICWSM).

11. Lampos, V., \& Cristianini, N. (2010). Tracking the flu pandemic by monitoring the Social Web. IAPR 2nd Workshop on Cognitive Information Processing. doi: 10.1109/CIP.2010.5604088.

12. Bollen, J., Mao, H., \& Zheng, X.J. (2011). Twitter mood predicts the stock market. Journal of Computational Science, 2(1), 1-8.

13. Sprenger, T.O., Welpe, I.M. (2010). Tweets and Trades - The Information Content of Stock Microblogs. Available at SSRN: http://ssrn.com/abstract=1702854.

14 Zhang, X., Fuehres, H., \& Gloor, P. (2010). Predicting Stock Market Indicators Through Twitter: "I hope it is not as bad as I fear". Available at: http://www.ickn.org/documents/COINs2010_Twitter4.pdf

15. Loewenstein,G.F., Weber, E.U., Hsee, C.K., \& Welch, N. (2001). Risk as Feeling. Psychological Bulletin, 127(2), 267-286.

16. Lerner, J., \& Keltner, D. (2001). Fear,Anger, and Risk. Journal of Personality and Social Psychology, 81(1), 146-159.

17. Dolan, R.J. (2002). Emotion, cognition, and behavior. Science, 298(5596), 1191-1194.

18. Lener, J.S., Small, D.A. \& Loewenstein, G. (2004). Heart strings and Purse Strings: Carryover Effects of Emotions on Economic Decisions. Psychological Science, 15, 337-341.

19. Shiv, B., Loewenstein, G., Bechara, A., Damasio, H., \& Damasio, A.R. (2005). Investment Behavior and the Negative Side of Emotion. Psychological Science, 16, 435-439. 